\title{
Heart is target of autoimmune attack after myocardial infarction in patients with T1DM
}

Myocardial infarction in people with type 1 diabetes mellitus (T1DM) leads to a 'runaway' autoimmune response that specifically targets cardiac tissue, a group of Harvard researchers suggest. The scientists demonstrated the persistent presence of autoantibodies against the cardiac tissuespecific protein myosin-6 (MyHC- $\alpha$ ) in the serum of patients with T1DM who have experienced such a cardiac event.

Cardiovascular disease is a major cause of death in people with T1DM and death following myocardial infarction is higher in patients with T1DM than in individuals without this disease. Despite substantial clinical advances that have contributed to reduce the incidence of several complications associated with T1DM, including renal failure and neuropathy, the rate of mortality owing to cardiovascular disease in these patients has not improved over the past three decades.

$\mathrm{MyHC}-\alpha$ is released at high levels from necrotic cardiac tissue and becomes an immune stimulus. The innate immune response is a key pathway involved in repairing damaged tissue. However, "the MyHC- $\alpha$ transcript is not expressed in the thymus of humans or mice, which is the first and most important place where T cells are 'trained' in recognizing the body's own cells so they refrain from attacking them," explains lead investigator Myra Lipes. Studies in nonobese diabetic mice showed that myocarditis following myocardial infarction is mediated primarily by pathogenic $\mathrm{CD} 4^{+} \mathrm{T}$ cells with MyHC- $\alpha$ as their main target.

"People could develop autoimmune reactions to heart muscle after inflammatory injury, such as myocardial infarction," explains Lipes. "In T1DM, these responses would become persistent and amplified-'runaway' responses."

In a small group of patients with T1DM $(n=18)$ aged $55 \pm 12$ years who had a history of myocardial infarction, two-thirds tested positive for MyHC- $\alpha$ autoantibodies 4.5 years on average (range $0.3-8$ years) after this cardiac event. In patients with type 2 diabetes mellitus (T2DM) who had experienced myocardial infarction $(n=20)$, only $10 \%$ tested positive for MyHC- $\alpha$ autoantibodies.

The researchers also showed that inducing expression of $\mathrm{MyHC}-\alpha$ in the thymus of diabetic mice

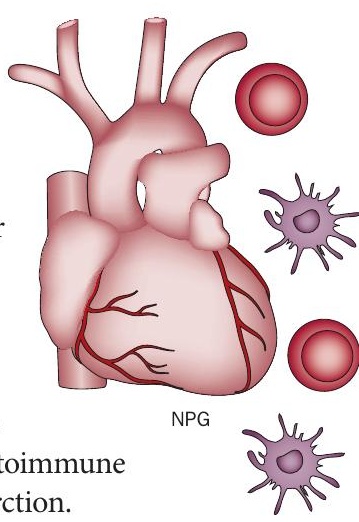
reaction to myocardial infarction.

Autoantibodies against MyHC-a were no longer detected and the damaged tissue was able to heal.

"Until now, the cardiovascular complications of T1DM and T2DM were lumped together and approached in the same way," says Lipes. "However, our findings suggest new ways to diagnose and treat heart disease in patients with T1DM through development of antigen-specific approaches."

\section{Fiona Mitchell}

Original article Gottumukkala, R. V. et al. Myocardial infarction triggers chronic cardiac autoimmunity in type 1 diabetes. Sci. Trans. Med. 4, 138ra80 (2012) 